\title{
11 Ästhetische Chirurgie des äußeren weiblichen Genitales
}

Simone Preiß

\subsection{Medizingeschichtlicher und kultureller Hintergrund}

Die Rasur der Schambehaarung, die bei den unter 30-jährigen Frauen und Männern heute als selbstverständlich gilt (Bravo Dr.-Sommer-Studie 2006), führt zu einer besseren Sichtbarkeit der äußeren weiblichen Genitalregion. Hierdurch werden Abweichungen vom ästhetischen Ideal von einigen Frauen als störend oder sogar inakzeptabel empfunden. Das heutige ästhetische Genitalideal orientiert sich im Bereich der Vulva der Frau an einem kindlichen Zustand (Lolita-Effekt) mit straffen, vollen, äußeren Schamlippen (Labia majora) und durch diese verdeckte, kleinere innere Schamlippen (Labia minora). Das Hervorschauen der Labia minora beim aufrechten Stand über das Niveau der Labia majora hinaus wird mit dem Verlust von Jugendlichkeit und einem verstärkten Sexualtrieb assoziiert. Solche Herabwürdigungen, des durch natürliche Alterungsprozesse, Schwangerschaften und Hormonschwankungen, Veränderungen unterworfenen weiblichen Geschlechts, basieren zum einen auf traditionellen Vorstellungen („Das Gemächt des Weibes liege innwendig, das des Mannes auswendig. " [Schenda 1998, S. 300]) und werden durch populistische und idealisierende Medien sowie die Pornoindustrie weiter manifestiert. Die Dr.-Sommer-Studie „Liebe! Körper! Sexualität!“ der Zeitschrift Bravo (2009) zeigt, dass nur 37\% der befragten Jugendlichen noch keinen Kontakt mit Pornografie hatten; 69\% aller Jungen und 57\% aller Mädchen haben schon pornografische Bilder oder Filme gesehen. Die Idealisierung des weiblichen Ge- 
nitales führt zu unrealistischen Vorstellungen - insbesondere von Jugendlichen - bezüglich des normalen Aussehens einer Vulva und der Veränderungen, dem dieser Körperteil während der Pubertät unterliegt. Mit der Orientierung an einer Idealform wächst die Unzufriedenheit mit dem Aussehen der eigenen Scham. Die Akzeptanz von chirurgischen Korrekturen steigt - auch mithilfe der Medien. Eine niederländische Untersuchung (Koning et al. 2009) zeigt, dass fast alle befragten Frauen erst in den letzten 2,2 \pm 2,2 Jahren von der Möglichkeit einer Reduktionsplastik der kleinen Labien gehört hatten. Als Informationsquellen wurden das Internet und Medien wie TV, Radio und Magazine genannt. Nicht zuletzt haben Reality-Shows die Nachfrage nach diesen Operationen gesteigert (Medalie 2010).

Der Wunsch nach einer Genitalkorrektur wird seit einigen Jahren auch in Deutschland immer häufiger an Gynäkologen und Plastische Chirurgen herangetragen.

Lehrbücher für Plastische Chirurgie behandeln das Thema ästhetische Genitalchirurgie bis 2010 nur marginal oder fokussieren auf Korrekturen des männlichen äußeren Genitales (Penisverlängerung, Korrektur der Penisdeviation). So widmet etwa das achtbändige themenübergreifende Gesamtwerk „Plastic Surgery“ von Mathes in der Ausgabe von 2006 der „,female genital cosmetic surgery" (Alter 2006, 404-409) gerade mal sechs Seiten verglichen mit 16 Seiten über die Probleme mit dem Aussehen des männlichen Cliedes. Die Operationstechniken entstammen einerseits Modifikationen von Rekonstruktionen von Vulva- und Vaginaldefekten nach Krankheit oder Trauma, andererseits aus der Fehlbildungschirurgie. Es werden die Verkleinerung der Labia minora beschrieben, Eigenfettinjektionen zum Auffüllen erschlaffter Labia majora und bei prominenter Klitoris erwähnt sowie Korrekturen bei Cutis laxa im Bereich des Schambeines durch untere Abdominoplastik sowie die Liposuktion bei störendem Fettpolster über dem Schambein. Eine Definition der notwendig vorliegenden Veränderungen der Schamlippen zur Stellung der Operationsindikation wird vermieden: zu groß oder zu klein ist hier, was die Patientin stört. In dem vierbändigen deutschen Standardwerk „Plastische Chirurgie“ von 2007 (Piza-Katzer u. Balogh 2007) wird die ästhetische Verkleinerung der Labia minora mit folgendem Satz abgehandelt:

„Eine Labioplastik unter ästhetischen Gesichtspunkten soll so geschehen, dass die Labia minora noch mindestens einen Zentimeter hoch bleiben und dass die Resektionslinie nicht zu nahe an das Orificium urethrae externum heranreicht." (Piza-Katzer u. Balogh 2007)

Alle weiteren Techniken zur ästhetischen Korrektur des weiblichen Genitales werden, bis auf die aus den islamischen Ländern stammende weibliche Form der Zirkumzision, nicht erwähnt.

Erst in dem 2010 veröffentlichten themenübergreifenden Gesamtwerk „Plastic and Reconstructive Surgery“ (Siemionow u. Eisenmann-Klein 2010) erfolgt 
eine ausführlichere Darstellung der ästhetischen Chirurgie des weiblichen Genitales und erstmals liefert Gress 2011 ein eigenes Kapitel mit dem Titel: „Form- und funktionsverbessernde Eingriffe im weiblichen Genitalbereich“ in einem deutschsprachigen Standardwerk zur Ästhetischen Chirurgie (Gress 2011) nach. Dies spiegelt die noch junge, aber aufgrund der steigenden Nachfrage notwendige Auseinandersetzung der Plastischen Chirurgen mit der ästhetischen Genitalchirurgie bei Frauen in den letzten Jahren wider.

\subsection{Ausprägungen und gängige Formen}

Während das Intimideal heute massenmedial erörtert und präsentiert wird, bleiben Anstrengungen zur Erforschung und die Vermittlung von Wissen über Form und Funktion vor allem der weiblichen Geschlechtsorgane dahinter zurück. So besteht selbst unter Gynäkologen eine erhebliche Unsicherheit bei der Definition der vergrößerten inneren Schamlippen, der Labienhypertrophie (Paarlberg u. Weijenborg 2008). Im Jahre 2005 zeigte die Vermessung von 50 prämenopausalen Frauen, dass es eine große Variationsbreite (Lloyd et al. 2005) hinsichtlich Form und Färbung des äußeren Genitales der Frau gibt. Der Altersdurchschnitt dieser Untersuchung lag bei 35,6 Jahren. Lloyd et al. (2005) fanden dabei beispielsweise Ausdehnungen der Labia minora zwischen Klitoris und Damm (Länge der kleinen Schamlippen) zwischen 20 und $100 \mathrm{~mm}$ (s. Tab. 1 und Abb. 1). Sie beschrieben Breiten der inneren Schamlippen zwischen 7 und $50 \mathrm{~mm}$, mit einer mittleren Breite von 21,8 mm. Auch die Länge der Klitoris und die Breite der Glans clitoridis waren sehr variabel. Große innere Schamlippen sind demzufolge eine Normvariante und nichts Abnormes. Die Formvariationen der äußeren weiblichen Geschlechtsorgane haben verschiedene Ursachen, sie können unter anderem auf anlagebedingte Gewebeeigenschaften und erbliche Faktoren zurückgeführt werden.

Da keine der Frauen dieser Erhebung Probleme beklagte (Lloyd et al. 2005) kann abgeleitet werden, dass eine echte Labienhypertrophie erst ab $5 \mathrm{~cm}$ Breite vorliegt. Eine Operationsindikation besteht aber auch nur dann, wenn Beschwerden vorliegen. Bei allen anderen Operationen zur Verkleinerung der inneren Schamlippen muss von einer kosmetischen Operation gesprochen werden (Liao u. Creighton 2007). Liegt eine Klitorishypertrophie vor, ist eine hormonelle und in manchen Fällen auch eine chromosomale Untersuchung zur Abklärung von intersexuellen Erscheinungsformen anzuraten.

\subsubsection{Anatomie der Vulva}

$\mathrm{Zu}$ den äußeren weiblichen Geschlechtsorganen gehören der Schamhügel (Mons pubis), die großen Schamlippen (Labia majora pudendi), die kleinen Schamlippen (Labia minora pudendi), der Scheidenvorhof (Vestibulum vaginae), der Vorhofschwellkörper (Bulbus vestibuli), der Kitzler (Klitoris) und die 
11 Ästhetische Chirurgie des äußeren weiblichen Genitales

Tab. 1 Diversität der Labia minora (nach Lloyd et al. 2005, S. 643)

\begin{tabular}{|c|c|c|}
\hline Kriterium & Schwankungsbreite & $\begin{array}{c}\text { Mittelwert } \\
\text { (Standardabweichung) }\end{array}$ \\
\hline Klitorislänge (mm) & $5-35$ & $19,1(8,7)$ \\
\hline Breite der Glans clitoridis (mm) & $3-10$ & $5,5(1,7)$ \\
\hline Distanz Klitoris - Urethra (mm) & $16-45$ & $28,5(7,1)$ \\
\hline $\begin{array}{l}\text { Ausdehnung Labia majora zw. vorderer } \\
\text { und hinterer Kommisssur (Länge in } \mathrm{cm} \text { ) }\end{array}$ & $7,0-12,0$ & $9,3(1,3)$ \\
\hline $\begin{array}{l}\text { Ausdehnung Labia minora zw. vorderer } \\
\text { und hinterer Kommisssur (Länge in mm) }\end{array}$ & $20-100$ & $60,6(17,2)$ \\
\hline Breite der Labia minora (mm) & $7-50$ & $21,8(9,4)$ \\
\hline Länge Vagina (cm) & $6,5-12,5$ & $9,6(1,5)$ \\
\hline Farbe Vulva (n) & & $n$ \\
\hline wie umgebende Haut & & 9 \\
\hline dunkler als umgebende Haut & & 41 \\
\hline Fältelung der Labien (n) & & $n$ \\
\hline glatt & & 14 \\
\hline mäßig & & 34 \\
\hline ausgeprägt & & 2 \\
\hline
\end{tabular}

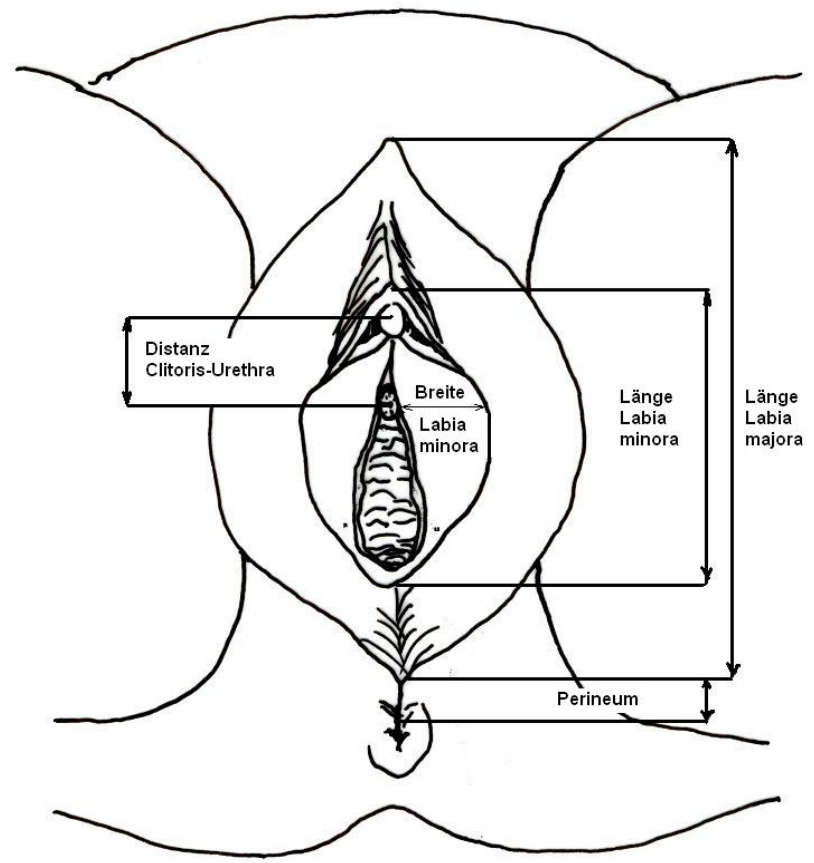

Abb. 1 Messbereiche (nach Lloyd et al. 2005) 
Vorhofdrüsen (Glandulae vestibulares), zu denen die Bartholin-Drüsen gehören (s. Abb. 2). Die äußeren weiblichen Geschlechtsorgane werden auch kurz Vulva genannt. Sie sind von den inneren Geschlechtsorganen durch den Hymen (sogenanntes Jungfernhäutchen) abgegrenzt. Ist der Hymen entfernt, hat man zwischen den kleinen Schamlippen Einsicht in den Scheidenvorhof. Dieser beherbergt auch die Mündung der Harnröhre (Ostium urethrae externum). Auf der Innenfläche der kleinen Schamlippen münden die paarigen großen Vorhofdrüsen (Glandulae vestibulares majores bzw. Bartholin-Drüsen). Gemeinsam mit den kleinen Vorhofdrüsen (Glandulae vestibulares minores) bilden sie ein schleimiges Sekret, das den Scheidenvorhof befeuchtet, dadurch beim Geschlechtsverkehr die Reibung vermindert und somit Epithelschäden vorbeugt. Ein Verschluss der Ausführungsgänge der großen Vorhofdrüsen durch eine Entzündung oder Vernarbung kann zur Ausbildung von Retentionszysten führen, die stark schmerzhaft sind und der operativen Entfernung bedürfen.

Die Schamlippen vereinigen sich am Mons pubis in einer vorderen Kommissur, welche die Klitoris umschließt. Von der Klitoris aus strahlen zwei Hautfalten in die kleinen Schamlippen ein, die Frenula clitoridis.

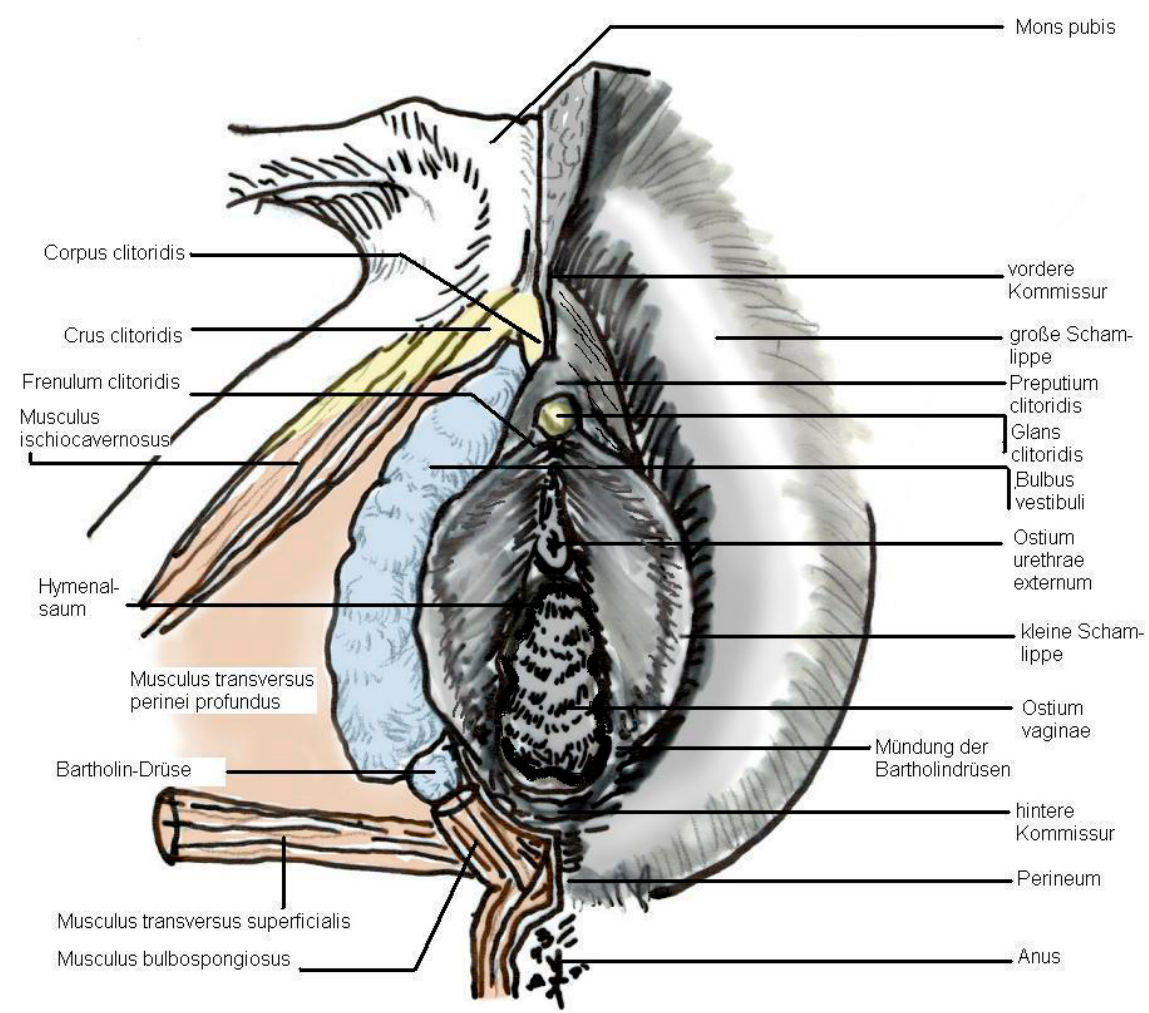

Abb. 2 Vulva, auf der linken Bildhälfte ist die Haut entfernt 
Die Klitoris besteht nicht nur aus der äußerlich sichtbaren Glans clitoridis, sondern auch aus einem Klitorisschaft (Corpus clitoridis), der von der kapuzenartig aufsitzenden Klitorisvorhaut (Praeputium clitoridis) bedeckt ist. Um den Klitorisschaft und den seitlich unter dem Musculus ischiocavernosus verlaufenden Klitorisschenkel (Crura clitoridis) herum befinden sich Schwellkörper (schwellfähiges Venengeflecht), die denen des männlichen Penis entsprechen. Schwellkörpergewebe findet sich auch in der Basis der Schamlippen (Bulbus vestibuli). Der Bulbus vestibuli wird vom Musculus bulbospongiosus bedeckt.

Die Vulva weist eine ausgesprochen gute Blutversorgung auf. Aus Ästen der Arteria pudenda interna werden der Anus, die Dammregion, die Schwellkörpermuskeln sowie der hintere Teil der großen Schamlippen (Rami labiales posteriores aus der Arteria perinealis) mit arteriellem Blut versorgt. Der vordere Teil der großen Schamlippen wird über Äste der Arteria femoralis (Rami labiales anteriores aus den Arteriae pudendae externae) versorgt. In den großen Labien anastomisieren die von vorn und hinten kommenden Gefäßäste (Hwang et al. 1983). Diese Gefäßnetze geben an die kleinen Labien wiederum multiple Gefäßbögen ab. Der venöse Abstrom des Blutes erfolgt analog über die Vena pudenda interna, mehrere Venae pudendae externae und zusätzlich in den Plexus venosus vesicalis.

Die vorderen Anteile der großen Schamlippen werden sensibel von Nervenästen aus dem Nervus ilioinguinalis (Plexus lumbalis, L1-L4) versorgt. Der hintere Anteil der Schamlippen, die Dammregion sowie die Haut des Anus innervieren oberflächliche Äste des Nervus pudendus aus dem Plexus sacralis (L5S3). Tiefe Äste des Nervus pudendus innervieren auch die Schwellkörpermuskeln und die Klitoris. Die Klitoris weist bis zu 8.ooo Nervenendigungen auf.

\subsubsection{Operationstechniken zur Reduktion der kleinen Schamlippen}

Die Verkleinerung der Labia minora ist die am häufigsten durchgeführte Korrekturoperation am weiblichen Genitale (Labiaplasty) (s. Abb. 3). Bereits 1739 veröffentlicht der deutsche Militärchirurg Lorenz Heister (1683-1758) in „Institutiones Chirurgicae“ (Ricci 1949) die Behandlung einer exzessiv hypertrophierten Klitoris und übergroßer, symptomatischer Labia minora. Bisher existiert keine Standardoperationstechnik zur Verkleinerung der inneren Schamlippen. Daher kommen zurzeit verschiedene Techniken zur Anwendung, wobei für einige Techniken bisher nur Fallstudien veröffentlicht wurden. Die wissenschaftlichen Veröffentlichungen zum Thema haben seit dem Jahr 2001 an Fahrt aufgenommen - so wurden zwischen 1976 und 2000 sechs, von 2001 bis 2005 bereits neun und zwischen 2006 bis 2009 weitere 25 wissenschaftliche Arbeiten auf Englisch publiziert (Liao u. Creighton 2007). 

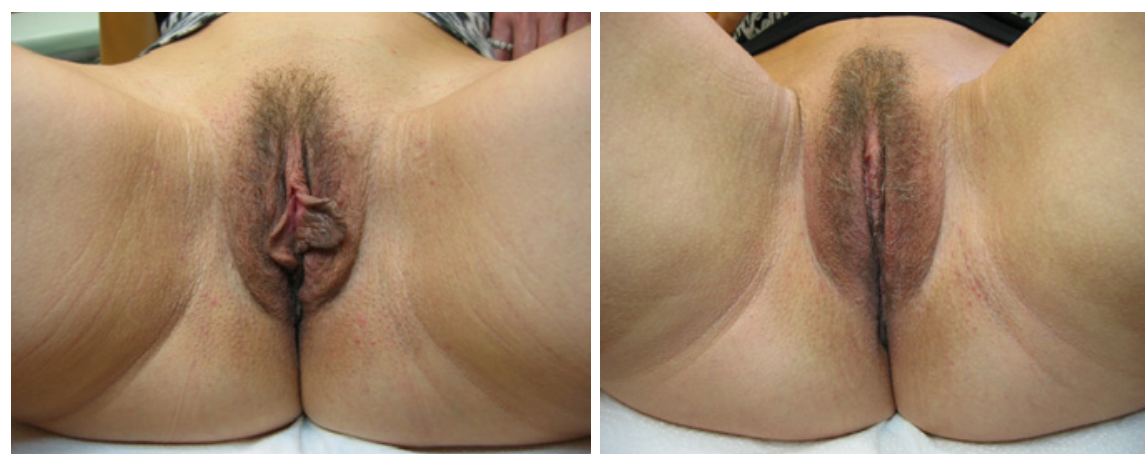

Abb. 3 Labienverkleinerung (mit freundlicher Genehmigung von Dr. med. Marwan Nuwayhid, LANUWA AESTHETIK, Leipzig)

\subsubsection{Zentrale Keilexzision nach Alter}

Hinsichtlich der Wahl des Operationsverfahrens zur Verkleinerung der kleinen Schamlippen hebt Alter (2006) die Bedeutung des Erhaltes der normalen Anatomie, einschließlich des Labienrandes hervor. Als Basisoperation schlägt er daher die V-förmige Exzision des am meisten elongierten Labienanteils vor. Die von Alter 1998 ( $n=4)$ vorgestellte Technik zeichnet sich durch eine Gewebereduktion durch zentrale Keilexzision im Bereich der kleinen Labien aus. Dabei wird über der größten Labienbreite eine V-förmige Exzisionsfigur angezeichnet, deren Spitze an der Innenseite der kleinen Schamlippen bis in den Introitus vaginae reicht (s. Abb. 4). Nach Infiltration eines Lokalanästhetikums erfolgt die Keilexzision zunächst an der kleineren der beiden Labia minora. Danach wird angleichend der Gewebeüberschuss der zweiten kleinen Schamlippe exzidiert.

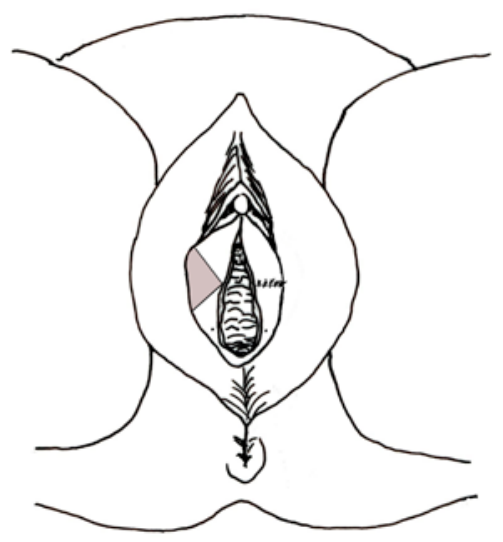

Abb. 4 Zentrale Keilexzision; linke Bildhälfte mit angezeichneter Schnittführung, rechte Bildhälfte nach Wundverschluss 
Mit dieser Technik kann eine randständige Narbe durch Erhalt der originären Labienkante vermieden werden. Die spezifische Kontur und Pigmentierung der kleinen Schamlippen bleibt somit bestehen. Jedoch wird die neurovaskuläre Versorgung am Labienrand unterbrochen. Nachteilig erscheint auch, dass die zentrale Keilexzision bei großen Labia minora nur eine ungenügende Gewebereduktion zulässt und keine Reduktion in der Periklitoralregion (Ellsworth et al. 2010) erlaubt. Es kann zu Wundheilungsstörungen mit einem Auseinanderweichen der Wundränder kommen (Hohl et al. 2008). Ferner sind Verengungen des Introitus vaginae durch die Narbenbildung in der wissenschaftlichen Literatur beschrieben (Giraldo et al. 2004; Solanki et al. 2009). Der senkrecht auf den Scheideneingang zulaufende Narbenzug kann zu ziehenden Schmerzen beim Geschlechtsverkehr führen.

\subsubsection{Erweiterte zentrale Keilexzision nach Alter}

Um die Nachteile der einfachen Keilexzision zu eliminieren, stellte Alter 2006 eine Erweiterung der zuvor beschriebenen Technik, durch eine an der Außenseite der kleinen Labien abweichende Schnittführung, vor $(\mathrm{n}=407)$. Das äußere „V“ wird dabei bogenförmig bis kurz vor die vordere Kommissur verlängert (sog. Hockey-Stock-Exzision). In der Verlängerung des „V“ wird an den Außenseiten der kleinen Schamlippen die Haut deepithelisiert und damit eine laterale Resektion des überschüssigen kapuzenartigen Gewebes um die Klitoris herum erreicht (lateral hood reduction). Alternativ kann Letzteres auch durch eine separate spindelförmige Resektion des vorderen Labienanteils erfolgen, wobei die entstehende Narbe jedoch auf der Labienkante zu liegen kommt (medial hood reduction) und ein Farbunterschied am vorderen und hinteren Labienanteil auftreten kann.

Gegenüber der einfachen zentralen Keilexzision erlaubt diese erweiterte Technik eine vermehrte Gewebereduktion der kleinen Labien und schließt die Periklitoralregion mit ein.

\subsubsection{Zentrale Deepithelialisierung nach Choi und Kim}

Je nach Größe der kleinen Labien wird im zentralen Labienanteil sowohl an der Innen- als auch an der Außenseite das zu resezierende Areal ovaluär angezeichnet (Choi u. Kim 200o; $n=6$ ). Nach Unterspritzen eines Lokalanästhetikums wird nun die angezeichnete Fläche deepithelisiert. Anschließend werden die Wundränder einander angenähert und vernäht (s. Abb. 5). Diese Technik erlaubt den Erhalt der neurovaskulären Versorgung am Labienrand sowie von natürlicher Textur und Farbe der kleinen Labien. Ferner wird eine Narbe auf der Labienkante vermieden. Jedoch ist die Gewebereduktion bei Schamlippen von 4 bis $6 \mathrm{~cm}$ Breite und bei sehr langen Schamlippen häufig unzureichend (Ellsworth et al. 200o). Nachteilig ist auch, dass die präparatorisch an- 
spruchsvolle Deepithelialisierung häufig eine verdickte Labia minora aufgrund des verbleibenden Labienparenchyms sowie einen Weichteilüberschuss in der Periklitoralregion hinterlässt.

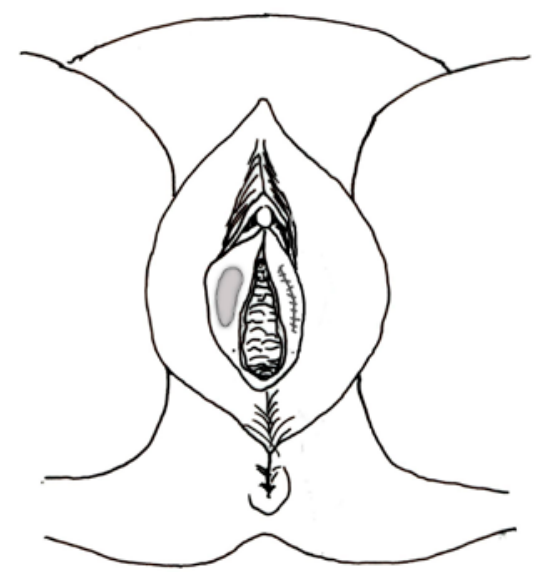

Abb. 5 Zentrale Deepithelisierung; linke Bildhälfte mit angezeichneter Schnittführung, rechte Bildhälfte nach Wundverschluss

\subsubsection{Inferiore Keilresektion und Rekonstruktion mittels superior gestielter Lappenplastik nach Rouzier et al. bzw. nach Munhoz}

Rouzier et al. $(n=163)$ stellten 2000 eine Technik vor, die sich insbesondere für lange, laxe Labia minora eignet. Dorsal des Übergangs des Frenulum clitoridis in die kleinen Labien wird dabei eine senkrechte Resektionslinie in Richtung der Labienbasis markiert (s. Abb. 6). Im Winkel von go Grad, parallel zur Lap-

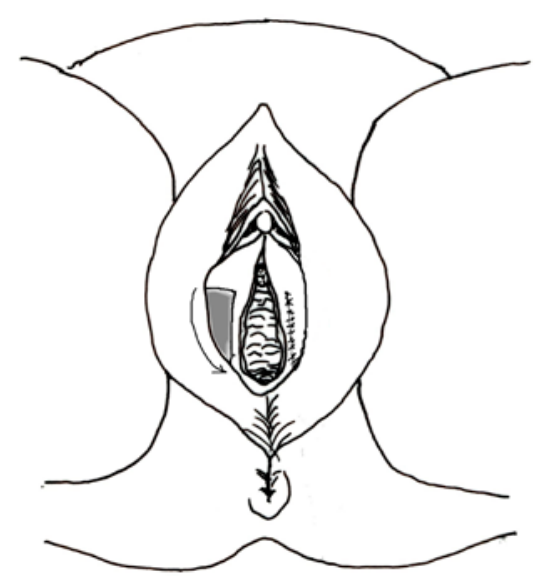

Abb. 6 Inferiore Keilexzision; linke Bildhälfte mit angezeichneter Schnittführung, rechte Bildhälfte nach Wundverschluss 
penbasis, verläuft hiervon ausgehend die weitere Resektionslinie in Richtung der hinteren Kommissur. Nach Exzision der angezeichneten Resektionsfigur wird der anteriore Lappen in den entstandenen Defekt eingenäht und bildet die neue Labienkante. Bei der Lappenplastik nach Rouzier et al. (2000) gilt zu beachten, dass das Verhältnis Lappenbreite zu -höhe von 1:2 nicht überschritten werden darf, da hier ein superior gestielter, randomisierter Lappen transponiert wird. Wird dies übersehen, sind Nekrosen im Bereich der Lappenspitze und Wundheilungsstörungen möglich. Wundheilungsstörungen traten bei $7 \%$ der operierten Frauen auf und waren mit Narbenkontrakturen, Asymmetrien und Dyspareunie assoziiert (Rouzier et al. 20oo). Raucher und Diabetiker haben ein höheres Risiko für Wundheilungsstörungen. Bei diesen Patienten sollte die Indikation zu dieser und der folgenden Operationstechnik sehr eng gestellt werden (Ellsworth et al. 2010).

Munhoz (2006, $\mathrm{n}=21$ ) bestimmt die Resektionsfigur im Bereich der kleinen Labien, indem er mit einer schmalen Pinzette den mittleren Anteil der kleinen Labie greift und diesen bis zum hinteren Anteil des Introitus vaginae (Punkt B) zieht. Die spätere Lappenspitze bildet der Punkt des originären Labienrandes, der Punkt B spannungsfrei erreicht und wird als Punkt A markiert. Das Anzeichnen des Exzisionskeiles erfolgt dann analog der Technik von Rouzier et al. (2000). Auch hier kommt die hintere Schnittkante nah am Introitus vaginae zu liegen, was zum Verlust von Drüsenausführungsgängen mit konsekutiver Scheidentrockenheit führen kann. Häufig wird auch der verbleibende Gewebeüberschuss im Bereich der Spitze des Resektionskeils als störend empfunden.

\subsubsection{Zentrale Keilexzision mit $90^{\circ}$-Z-Plastik nach Giraldo et al.}

Giraldo et al. (2004, $\mathrm{n}=15$ ) stellten 2004 eine Modifikation der bereits von Alter (Alter 1998) eingeführten einfachen Keilexzision im zentralen Drittel der kleinen Schamlippen vor. Erweitert wird diese Technik durch zwei go-gradige Abwinklungen, welche jeden Schenkel des „V“ zu einem „Z“ werden lassen (s. Abb. 7). Hierdurch soll neben der Breite der Labien auch deren Länge etwas vermindert werden und Narbenkontrakturen durch den unterbrochenen Narbenverlauf vorgebeugt werden. Wie bei der einfachen Keilexzision (Alter 1998) besteht jedoch das Risiko der Nahtdehiszenz (Hohl et al. 2008) und der ungenügenden Reduktion der Breite der kleinen Labien. Ferner kann die Unterbrechung der neurovaskulären Versorgung am Labienrand zu Sensibilitätsstörungen führen.

\subsubsection{Lineare Resektion der Ränder der kleinen Labien nach Caparo, modifiziert durch Turkof et al.}

Die Verkleinerung der kleinen inneren Schamlippen durch Entfernung eines Streifens entlang der gesamten Schamlippenkante wurde bereits 1971 durch 


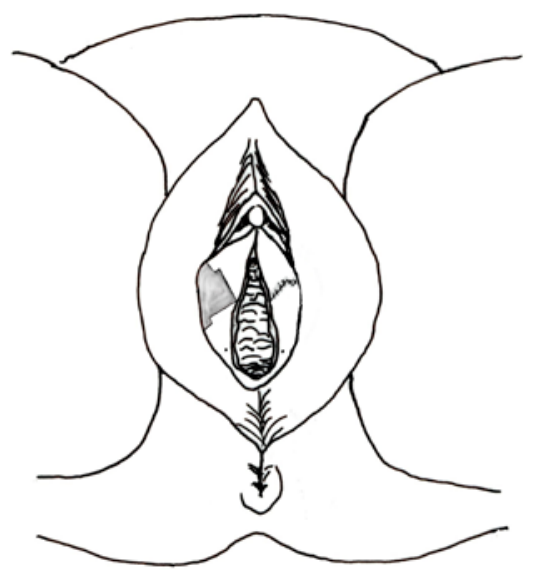

Abb. 7 Zentrale Keilexzision mit $90^{\circ}$ Z-Plastik; linke Bildhälfte mit angezeichneter Schnittführung, rechte Bildhälfte nach Wundverschluss

Caparo beschrieben und durch verschiedene Autoren, wie Turkof et al. (2008, keine Angabe zur Stichprobengröße) modifiziert. Nach Turkof et al. wird entsprechend des Wunsches der Patientin ein mehr oder weniger breiter Labiensaum belassen (s. Abb. 8). Dieser sollte mindestens o,8-1 cm breit sein, um eine Überkorrektur zu vermeiden. Beide Labia minora werden mit möglichst gleicher Spannung angehoben, die Resektionslinie markiert und der Überschuss entfernt. Verbleibende Asymmetrien können mit dieser Technik nicht immer ausgeschlossen werden. Auch eine vollständige Entfernung der inneren Schamlippen ist möglich, die laut Turkof et al. von der überwiegenden Mehrheit der Patientinnen gewünscht wird. Bei dieser einfach durchzuführenden Technik geht die in den meisten Fällen etwas dunkler gefärbte origi-

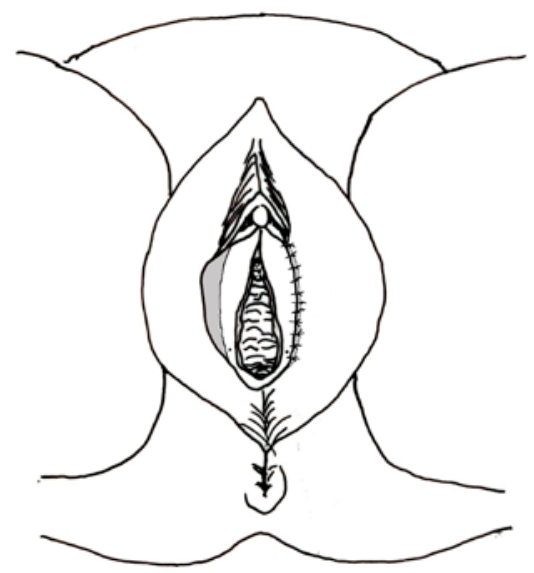

Abb. 8 Lineare Resektion; linke Bildhälfte mit angezeichneter Schnittführung, rechte Bildhälfte nach Wundverschluss 
näre Labienkante verloren. Dies wird von einigen Patientinnen gern in Kauf genommen, da mit der dunkleren Farbgebung der Labienkante häufig fortgeschrittenes Alter assoziiert wird (Ellsworth et al. 2010). Ein Nachteil der linearen Labienresektion besteht darin, dass die entstehende gerade Narbe auf der Labienkante das Risiko für Narbenkontrakturen und einer Dyspareunie verstärkt. Die Narbe kann dazu bei sportlichen Aktivitäten schmerzhaft sein (Alter 1998). Zudem verbleibt auch hier ein Gewebeüberschuss periklitoral.

\subsubsection{Laserreduktion der kleinen Schamlippen nach Pardo et al.}

Analog zur Linearen Resektion der Ränder der kleinen Labien nach Turkof et al. (2008) wird hier mittels Nd:YAG-Laser bei einer Leistung von $10 \mathrm{~W}$ das überschüssige Gewebe abgetragen (Pardo et al. 2006; $n=55$ ). Mit dieser Technik kann sowohl der zentrale Labienanteil als auch der periklitorale Labienanteil reduziert werden. Gegenüber der Reduktionsplastik mittels Skalpell ist der intraoperative Blutverlust durch die karbonisierende Laserwirkung des Nd:YAG-Lasers deutlich vermindert. Durch das für die ablative Laserchirurgie typische schichtweise Vorgehen, kann die Gefahr einer Überkorrektur minimiert werden und eine weitgehende Symmetrie erzielt werden. Die Wundflächen im Bereich der Labienkanten werden anschließend mittels Naht verschlossen. Jedoch wird auch hier eine Narbe auf der Labienkante in Kauf genommen und die originäre Hyperpigmentierung entfernt.

\subsubsection{Lineare Resektion der Ränder der kleinen Labien mit Einschluss des Frenulum und Praeputium clitoridis nach Gress}

Gress stellte 2007 ( $\mathrm{n}=279$ ) eine Modifikation der linearen Labienrandresektion vor, die auch die Periklitoralregion mit einschließt. Über die gesamte Länge der kleinen Labien verläuft die Schnittführung doppelt bogenförmig - der natürlichen Figur folgend - wobei ein Labienrest von o,8-1,o cm Höhe belassen wird (s. Abb. 9). Das kaudal in die Klitoris einstrahlende Gewebe (Frenulum clitoridis) wird ebenfalls bogenförmig reseziert. Wichtig ist hierbei nicht nur die Entfernung des Hautüberschusses unterhalb der Klitoris, sondern auch im Bereich der Klitoris selbst und darüber (Praeputium clitoridis), wofür der Schnitt entlang der Falte zwischen großer und kleiner Schamlippe fortgeführt wird (lateral clitoral hood reduction). Durch die Schnittführung entlang der gesamten Längsausdehnung der kleinen Labien kann eine maximale Gewebereduktion erreicht werden. Die hintere Schnittkante liegt jedoch nah am Introitus vaginae, was zum Verlust von Drüsenausführungsgängen führen kann, da hier die Bartolindrüsen münden (s. Abb. 2). Die große Wundfläche erhöht zudem die Gefahr von Wundheilungsstörungen. Da auch bei dieser Technik eine lineare, auf der zentralen Labienkante befindliche Narbe resultiert, besteht das Risiko für die Ausbildung von Narbenkontrakturen. 


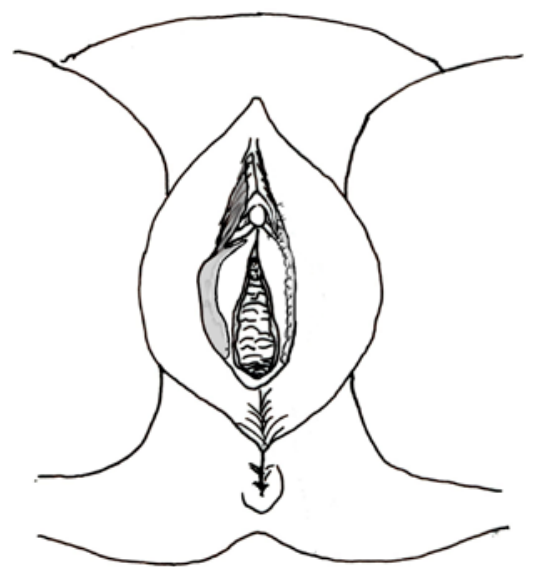

Abb. 9 Lineare Labienrandresektion mit Frenulumeinschluss; linke Bildhälfte mit angezeichneter Schnittführung, rechte Bildhälfte nach Wundverschluss

\subsubsection{Zentrale fortlaufend W-förmige Resektion nach Maas und Hage}

Um das Risiko der Narbenstrangbildung zu minimieren, wählen Maas und Hage (200o; $n=13$ ) eine Schnittführung, die den linearen Narbenverlauf entlang der Labienkante mehrfach unterbricht. Beginnend nach dem Übergang des Frenulum clitoridis in die kleinen Labien und vor der hinteren Kommissur endend, wird eine fortlaufend W-förmige Resektionsfigur angezeichnet, die auf der Innenseite der kleinen Labie gegenläufig zur Resektionsfigur der Außenseite verläuft (s. Abb. 1o). Nach Infiltration eines Lokalanästhetikums erfolgt die Gewebeexzision. Anschließend werden die medialen mit den late-

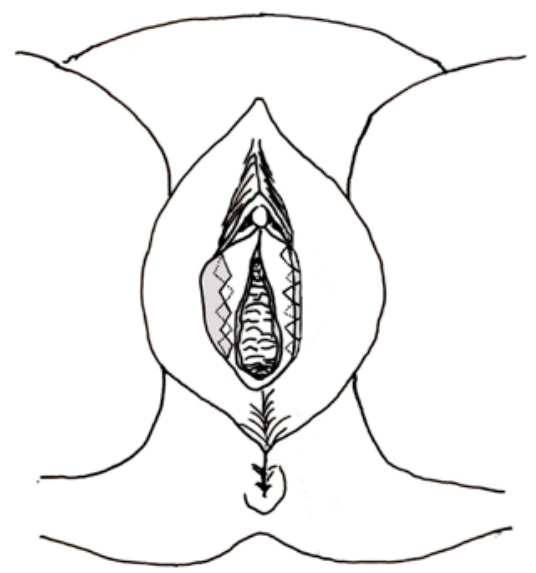

Abb. 10 Zentrale fortlaufend W-förmige Resektion; linke Bildhälfte mit angezeichneter Schnittführung, rechte Bildhälfte nach Wundverschluss 
ralen Läppchen mehrschichtig vernäht. Diese sehr aufwändige Technik ist mit einem erhöhten Risiko für Wundheilungsstörungen und Läppchennekrosen verbunden. Ferner kann die auf der Labienkante entstehende Narbe sehr unruhig durch die wechselnde Pigmentierung der einzelnen Läppchen erscheinen. Periklitoral und im Bereich der hinteren Kommissur kann ein ästhetisch störender Gewebeüberschuss verbleiben. Dieser minimiert jedoch andererseits das Risiko für Störungen der Sexualfunktion und der Empfindsamkeit (Solanki et al. 2009).

\subsubsection{Weitere Operationstechniken zur ästhetischen Verbesserung der weiblichen Genitalregion}

Neben den Reduktionsplastiken der kleinen Labien werden weitere chirurgische Veränderung der weiblichen Genitalien, die unter dem Begriff Female Genital Cosmetic Surgery (FCCS) subsumiert werden, wie die Verkleinerung oder Auffüllung erschlaffter großer Labien, angeboten. Da diese Eingriffe deutlich seltener durchgeführt werden, werden sie hier nur kurz erklärt.

\section{Verkleinerung der großen, äußeren Schamlippen und des Mons pubis}

Die Verkleinerung der großen, äußeren Schamlippen kann über eine spindelförmige Schnittführung erfolgen, wobei der Hautüberschuss über die gesamte Länge entfernt wird. Auch eine Fettabsaugung (Liposuktion) ist möglich oder die Kombination beider Techniken. Voluminöse Schamhügel können ebenfalls durch eine Liposuktion verkleinert werden (s. Abb. 11).
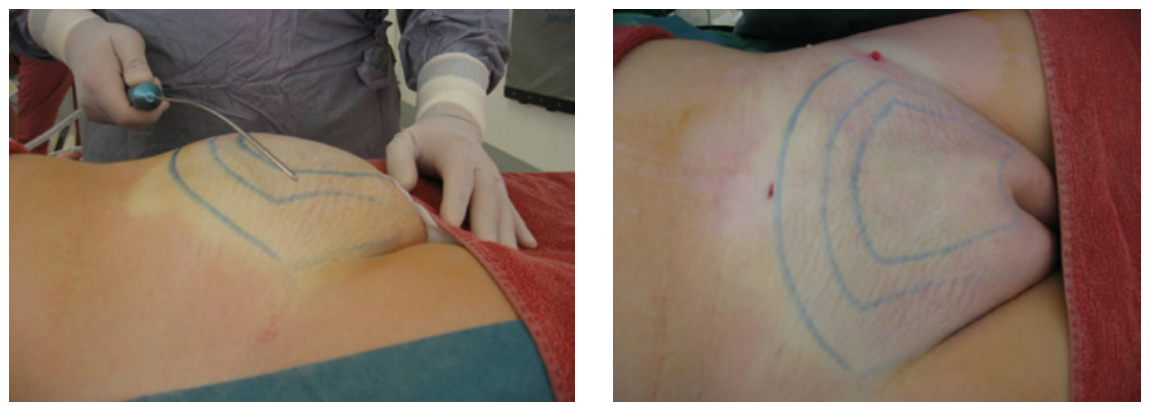

Abb. 11 Liposuktion Mons pubis (mit freundlicher Genehmigung von Dr. med. Marwan Nuwayhid, LANUWA AESTHETIK, Leipzig)

\section{Vergrößerung der großen, äußeren Schamlippen}

Zum Auffüllen altersbedingt erschlaffter oder gering ausgebildeter äußerer Schamlippen wird die Transplantation eigenen Fettgewebes, das zuvor an anderer Stelle abgesaugt und speziell aufbereitet wird, oder die Einbringung von Hyaluronsäure angewendet. Beide Techniken erbringen häufig keine dauer- 
hafte Auffüllung, da verpflanzte Fettzellen zum Teil und Hyaluronsäure vollständig vom Körper abgebaut wird und bedürfen daher mehrerer Wiederholungseingriffe.

\section{Verkleinerung oder Verlagerung der Klitoris}

Des Weiteren, häufig kombiniert mit der Labienreduktion, werden Eingriffe zur Verlagerung der Klitoris in Richtung Vaginaleingang und das sog. „clitoral unhooding“ - das Freilegen der Klitoris durch Entfernung oder Verkürzung ihrer kapuzenartigen Vorhaut - angeboten. Diese sollen der besseren sexuellen Stimulierbarkeit dienen. Um dies zu gewährleisten, müssen die sensiblen Nerven und die Gefäße, die in die Klitoris ziehen, unbedingt geschont werden, ansonsten droht ein Gefühlsverlust.

Das „clitoral unhooding“ wurde bereits vor gut hundert Jahren praktiziert. Lenzen-Schulte (2009) verweist auf den Bedeutungswandel, den dieser Eingriff in der Zwischenzeit erfahren hat:

„Wird dieser Eingriffheute zur Steigerung des Lustempfindens den Frauen angepriesen, diente er seinerzeit einem ganz anderen Zweck. Man wollte damit die Neigung der Frauen zur Masturbation eindämmen und ihr Sexualempfinden ,normalisieren': Die Frau sollte sich einzig durch den Geschlechtsverkehr mit einem Ehemann stimuliert fühlen."

Gress (2007) beobachtete eine zumeist unveränderte sexuelle Stimulierbarkeit der Klitoris nach „clitoral unhooding“. Nur 9\% der Frauen, die sich einer Reduktion der kleinen Labien mit periklitoraler Straffung unterzogen, beschrieben diesbezüglich eine Verbesserung. Eine Verschlechterung der Klitorissensibilität trat nicht ein.

Eine wirkliche Verkleinerung der Klitoris wird bei Anomalien ihrer Cröße und Form (echte Clitorishyperplasie) durchgeführt, wie sie bei der Intersexualität (uneindeutige Ausprägung des Geschlechtes) auftreten können. Bei der operativen Behandlung der Klitorishypertrophie wird der Gewebeüberschuss nach Deepithelisierung eines zirkulären Streifens an der Basis der Glans clitoridis unter das Hautniveau versenkt.

\section{Vergrößerung der G-Punkt-Region und Vaginalverengung}

Der Steigerung des Lustempfindens dienen auch Eingriffe im Bereich der inneren weiblichen Geschlechtsorgane, wie die G-Punkt-Unterspritzung mit Hyaluronsäure oder eigenen Fettzellen sowie Straffungsoperationen an der Vagina. Die Straffung einer als zu weit empfundenen Vagina (sog. Vaginalverengung, Vaginoplasty oder vaginal rejuvenation) nehmen vor allem Frauen in Anspruch, deren Vagina infolge von Geburten und altersbedingt an Muskeltonus verloren hat. Überflüssige Haut und Schleimhaut kann mittels oberflächlich abtragender Laser abgetragen werden, da dies zu einer Hauterneue- 
rung und -schrumpfung führt. Häufig ist die alleinige Straffung der Vaginalhaut jedoch nicht ausreichend, um eine dauerhafte Verkleinerung der Vagina zu erreichen. Daher kann die Verengung der Vagina auch durch eine Straffung der an die Vaginalhinterwand angrenzenden Rectusmuskelfaszie und eine Medialisierung der medialen Bäuche des M. levator ani ergänzt werden (hintere Kolporraphie, Gress 2011). Zusätzlich wird in die seitliche und vordere Vaginalwand (oder als alleinige Maßnahme zirkulär) eine Eigenfettunterspritzung durchgeführt. Dabei wird der Frau körpereigenes Fettgewebe aus dem Oberschenkel, dem Bauch oder Gesäß entnommen, um dieses nach einer speziellen Aufbereitung in den Vaginalbereich zu injizieren.

Bei der Vaginalverengung durch hintere Kolporraphie besteht eine anatomische Nähe zum Rektum und damit die Gefahr der Verletzung. Ferner müssen Überkorrekturen vermieden werden. Gress (2011) schlägt diesbezüglich vor, die Weite des Introitus vaginae für 2 Finger passend zu gestalten. Postoperativ wird durch einige Operateure ein Blasenkatheter eingelegt. Geschlechtsverkehr ist für mindestens $4-6$ Wochen zu vermeiden.

\section{Rekonstruktion des Hymens}

Vor allem in den Niederlanden, aber auch zunehmend von deutschen Chirurgen werden Jungfernhäutchenrekonstruktionen angeboten - die sogenannte, jedoch keineswegs standardisierte „Hymenorrhaphie“. Die biologische Funktion des Hymens ist nicht geklärt. Die Hymenrekonstruktion geschieht allein aus psychosozialen Gründen (Depression, Einsamkeit, Identitätskonflikte oder sogar Suizidalität). Meist wird sie von traditionell muslimischen Frauen angestrebt, um den Beweis der „Jungfräulichkeit“ zu erbringen, obwohl es nur bei 50\% aller Frauen überhaupt zu einer Blutung beim ersten Geschlechtsverkehr kommt (Wild et al. 2009).

Bei der dabei am häufigsten angewandten Operationstechnik werden die Kerben im gerissenen Hymenalsaum mit einen Skalpell oder einem Hochfrequenz-Radiochirurgiegerät angefrischt und anschließend mit selbst auflösendem Nahtmaterial fein vernäht (Gress 2011). Die Operation wird ca. 6 Wochen vor dem geplanten Beischlaf durchgeführt. Sind keine Hymenreste mehr vorhanden, kann ein zerreißbares Biomaterial am Introitus vaginae eingebracht werden, das durch Reibung und Druck zerstört wird und dann eine rote Flüssigkeit freigibt. Dieser Eingriff ist bis wenige Tage vor der vorgetäuschten Deflorierung möglich (Wild et al. 2009).

\subsection{Häufigkeiten}

Genaue Angaben über die Häufigkeit von Operationen im Rahmen der FGCS in Deutschland sind nicht verfügbar, da die Datenweitergabe an die die Häufigkeiten erhebende Fachgesellschaft (DGÄPC) nicht verpflichtend ist. Der be- 
kannteste deutsche Genitalchirurg Gress (2011) gibt an, zwischen 2003 und 2010 ca. 1.70o eigene Eingriffe im weiblichen Genitalbereich vorgenommen zu haben, davon ca. 60\% Reduktionsplastiken der Labia minora. Häufig werden Reduktionsplastiken der kleinen Labien in Kombination mit weiteren Eingriffen im Genitalbereich durchgeführt. So beschreibt Medalie (2010) bei den 118 von ihm zwischen 2005 und 2010 vorgenommen Reduktionsplastiken der Schamlippen in 13\% Kombinationen mit einer Vaginoplastik und in weiteren $24 \%$ Kombinationen mit anderen chirurgischen Eingriffen, wie Blasenaufhängung, Abdominoplastiken, Liposuktionen und Mammaaugmentationen.

Welche Technik bei der Verkleinerung der kleinen Schamlippen vorrangig eingesetzt wird, haben Mirzabeigi et al. (2012) unter US-amerikanischen Genitalchirurgen ermittelt. Meist kam die simple Amputation (52,7\%), gefolgt von der zentralen Keilexzision $(36,1 \%)$ zum Einsatz. Die zentrale Keilexzision mit ZPlastik wurde in $13,9 \%$ eingesetzt, W-förmige Randresektionen in $9,5 \%$ and S-förmige Randresektionen in 8,8\% (Mirzabeigi et al. 2012).

\subsection{Psychische Aspekte: Motive der Klienten}

Als Motive für eine Operation der Schamlippen werden vor allem lange innere Schamlippen und erschlaffte äußere Schamlippen sowie Asymmetrien der Schamlippen angegeben (Borkenhagen $u$. Kentenich 2009). Ästhetische Gründe, motiviert durch ausgeprägte Schamgefühle gegenüber dem eigenen äußeren Genitale, stehen also beim Wunsch nach einer Schamlippenreduktion im Vordergrund. Bei Vorliegen einer krankhaften Vergrößerung der Schamlippen können zusätzlich auch funktionelle Beeinträchtigungen, wie Einschränkungen bei der Kleiderwahl (Schmerzen beim Tragen enger Hosen), Einschränkungen beim Geschlechtsverkehr bzw. Urinieren (Invagination der inneren Labien oder Schmerzen), Einschränkungen bei Sport- und Freizeitaktivitäten (Schmerzen beim Radfahren, Wandern u.a.) und hygienische Aspekte zur Entscheidung zu einer operativen Korrektur beitragen (s. Abb. 12).

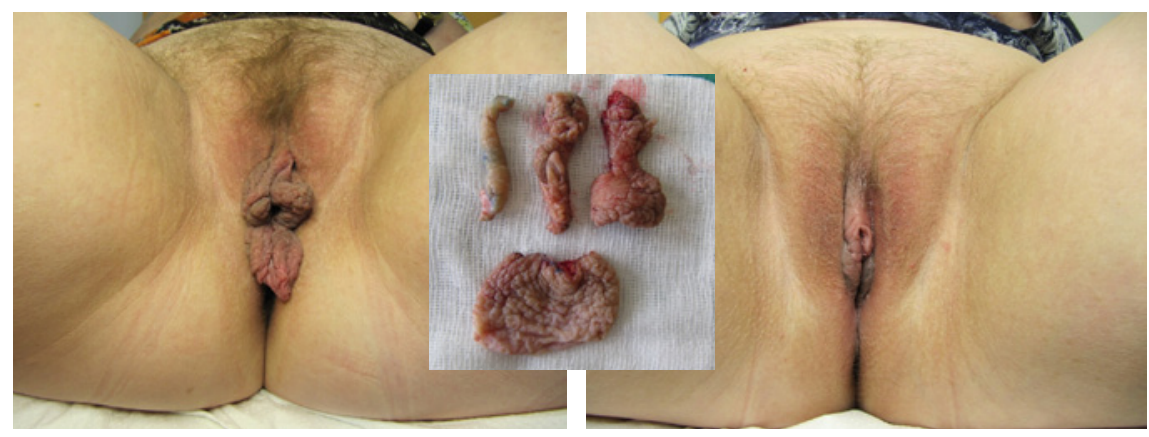

Abb. 12 Genitalästhetik (mit freundlicher Genehmigung von Dr. med. Marwan Nuwayhid, LANUWA AESTHETIK, Leipzig) 


\subsection{Umgang im medizinischen Alltag}

Die gesellschaftliche Debatte über die Genitalchirurgie, die in Deutschland 2008 entbrannte, wird bis heute kontrovers geführt. Aus chirurgischer Sicht werden besonders die Operationen am äußeren weiblichen Genitale als vergleichsweise simple und komplikationsarme Eingriffe dargestellt, die meist in Lokalanästhesie erfolgen. Die Operationszeiten betragen jedoch je nach Technik bis zu 2 Stunden und sind mit erheblichen Risiken behaftet (Gress 2011; Goodman 2011). Aus psychologischer Sicht kann die medial geschürte Unzufriedenheit mit dem Aussehen des eigenen Genitales durch Depressionen, narzisstische Störungen, Sexualstörungen oder Reifungskonflikte verstärkt werden oder diese überdecken (Borkenhagen et al. 2009). Die operative Therapie kann den psychischen Konflikt jedoch nicht lösen, ihn schlimmstenfalls sogar verstärken. Die in Österreich entwickelten Leitlinien zur Genitalchirurgie halten daher eine gynäkologisch-sexualmedizinische Anamnese für erforderlich, nicht zuletzt um eine „Body Dysmorphic Disorder“ auszuschließen. Dies setze sexualmedizinische und psychologische Grundkompetenzen der/ des behandelnden Ärztin/Arztes voraus (http://www. frauengesundheit-wien. at/frauengesundheit/weibliche_genitalchirurgie.html).

Die feministische Kritik bezieht sich auf die fehlende Abgrenzung zur rituellen Beschneidung von Frauen und sieht in der "freiwilligen Verstümmelung“ einen Beweis für die fortwährende Dominanz des „männlichen Blicks“ bei der Orientierung von Frauen an einem Schönheitsideal.

Untersuchungen, die die postoperative Zufriedenheit der Patientinnen erheben, sprechen eine eigene Sprache. Diese berichten je nach Prozedere in bis zu 97\% der Fälle von erfüllten Erwartungen und einer hohen selbsteingeschätzten postoperativen Zufriedenheit (ca. 85\%) der Patientinnen mit dem Operationsergebnis (Goodman 2010, $\mathrm{n}=285$ ). Die US-amerikanischen Plastischen Chirurgen schätzen die Zufriedenheit ihrer Patientinnen mit dem Operationsergebnis sogar noch höher ein (> 95\%) und zwar unabhängig von der gewählten Operationstechnik (Mirzabeigi et al. 2012, n = 2.255). Gress (2007) geht davon aus, dass die Zufriedenheit mit dem ästhetischen Ergebnis seit Erweiterung der Schamlippenreduktionsplastik auf den klitoralen und kranialen Schamlippenanteil noch gestiegen ist. Über eine Verbesserung des Lustempfindens berichten laut Goodman (2010) 64,7\% der Patientinnen mit Labienreduktionsplastik/,clitoral-unhooding“, 86,6\% der Patientinnen mit Vaginalverengung/ Perineoplastik und 92,8\% der Patienten mit kombinierten Eingriffen. Darüber hinaus haben 82,2\% der Patientinnen letzterer Cruppe das Gefühl, auch ihre Partner seien sexuell zufriedener (Goodman 2010). Gress (2007) fand keine Veränderungen der sexuellen Stimulierbarkeit in seinem Patientenkollektiv nach Korrekturen der Schamlippen und der Glans clitoridis. 


\subsection{Folgen und Gefahren}

Da es sich bei der ästhetischen Genitalchirurgie um ein relativ junges Feld der Plastischen Chirurgie handelt, besteht bei vielen Operateuren nur wenig Erfahrung und Training, wie sich auch an den veröffentlichten Fallzahlen der vorgestellten Operationstechniken zeigt. Gress (2011) berichtet, dass jede vierte Genitaloperation in seiner Praxis inzwischen eine Nachkorrektur sei. Er begründet dies damit, dass die Operation häufig unterschätzt werde. Bei den Nachkorrekturen handelt es sich vor allem um verbliebene Asymmetrien oder Nachkürzungen der inneren Labien bei Unterkorrektur. Bei falsch gewählter Operationstechnik kann ein unharmonisches Gesamtbild verbleiben, z.B. wenn bei einer Reduktion der kleinen Schamlippen die überschüssige Haut periklitoral nicht entfernt wird.

Komplikationen bei chirurgischen Maßnahmen werden in geringgradig (keine Revision notwendig), mittelgradig (kleine Revision notwendig oder verlängerter Heilungsverlauf 1 bis 3 Monate) und schwerwiegend (schwerwiegende revisionspflichtige Komplikation oder chronische Beschwerden) eingeteilt. Medalie (2010, $\mathrm{n}=118$ ) verzeichnete ausschließlich gering- bis mittelgradige Komplikationen, wie seltene Infektionen und lokale Stichkanalabszesse durch subkutane Vicrylfäden. Sieben Nachkorrekturen aufgrund von Asymmetrien, Unterkorrektur oder Narben werden berichtet. Der längste Heilungsverlauf mit Hautirritationen und Schmerzen beim Geschlechtsverkehr wurde mit vier Monaten angegeben. Weitere vier Patientinnen hatten solche Beschwerden bis drei Monate nach dem Eingriff (Medalie 2010). Gress (2007, $\mathrm{n}=364$ ) gibt 11 Fälle von Wundheilungsstörungen als häufigste Komplikationen an, darunter zwei revisionsbedürftige Nachblutungen. Nekrosen oder Infektionen kamen nicht vor. Asymmetrien wurden in 9 Fällen nachkorrigiert, Unterkorrekturen der kleinen Labien in 18 Fällen nachgekürzt.

Von extremer Bedeutung ist daher die Patientenaufklärung. So muss präoperativ klar gemacht werden, dass die erwünschte Verbesserung des Lustempfindens nicht garantiert werden kann und Narbenbeschwerden sogar zu einer Verschlechterung der Kohabitationsfähigkeit führen können. Das Risiko der Kosten für Wiederholungseingriffe, die z.B. bei der Eigenfetttransplantation und Komplikationen notwendig werden können, liegt allein bei der Patientin.

\subsection{Rechtliche bzw. gesetzliche Regelungen}

Operationen am weiblichen Genitale gelten als ästhetische Operationen, solange durch eine differenzierte Diagnostik angeborene oder erworbene Fehlbildungen bzw. Veränderungen der Vulva im Rahmen eines adrenogenitalen Syndroms, nach Trauma, Neoplasien oder anderen Krankheiten ausgeschlossen wurden. Da bisher keine einheitliche Definition einer Hypertrophie der Labia minora gefunden wurde, gilt Übergröße der kleinen Schamlippen als 
subjektive Einschätzung ohne Krankheitswert im Sinne der gesetzlichen Krankenversicherung. Der Medizinische Dienst der Krankenkassen (MdK) folgt hier der gleichen Argumentationslinie wie bei der Beurteilung der Mammahypertrophie, bei der häufig eine Normvariante angenommen wird, bei der eine operative Verkleinerung medizinisch nicht notwendig und sogar gefährlich sein kann (Breitmeier 2006). An privatärztliche Leistungen werden besonders strenge Forderungen hinsichtlich der Aufklärungspflicht des Arztes gegenüber dem Patienten gestellt. Da operative Standards, Risikoeinschätzungen und Komplikationsraten hinsichtlich der kosmetischen Operationen am weiblichen Genitale jedoch bisher fehlen (keine Nachuntersuchungen über längere Zeiträume), fordert die Deutsche Gesellschaft für Gynäkologie und Geburtshilfe e.V. in der „Stellungnahme der DGGG zur Intimchirurgie“ vom 13. Juli 2009 eine gründliche Aufklärung über mögliche Komplikationen und Risiken dieser Eingriffe, wobei das Auftreten von

„Sensibilitätsstörungen mit herabgesetzter sexueller Empfindlichkeit, veränderte taktile Empfindungen bis hin zu deutlichen Funktionsbeeinträchtigungen des Cenitale (z.B. Dyspareunie, nicht Eintreten der gewünschten Stimulationswiederherstellung)“

betont wird. Das American College of Obstetricians and Gynecologists hat folgende Empfehlungen herausgegeben:

1. Vor einem solchen Eingriff sollten die Motive für die Operation genau abgeklärt werden.

2. Es sollte ein körperlicher Befund für den Eingriff vorliegen.

3. Die Patientinnen müssen darüber aufgeklärt werden, dass bisher keine ausreichenden wissenschaftlichen Daten darüber vorliegen, dass diese Eingriffe zu anhaltenden psychischen oder funktionellen Verbesserungen führen.

4. Es muss über die Risiken der Eingriffe, wie Infektionen, veränderte Sensibilität, Dyspareunie, Verwachsungen und Narben umso detaillierter aufgeklärt werden, je weniger der Eingriff den Charakter einer ärztlichen Heilmaßnahme hat. Diese ausführliche Aufklärung muss sorgfältig dokumentiert werden.

5. Es muss darauf hingewiesen werden, dass es für diese Operationen keine wissenschaftlich erarbeiteten Operationsstandards gibt, die bei unzureichenden Operationsergebnissen als Klagegrund verwendbar wären.

\subsection{Fazit für den Umgang im klinischen Alltag}

Bei Operateuren mit spezifischer Expertise und einem vorausgegangenen Training für diese Eingriffe sowie genauen Kenntnissen der Anatomie des weiblichen Genitales sind operativ bedingte Komplikationen selten (Goodman 2009; DGCG). Die Entwicklung von Leitlinien für vaginale und pelvine Ein- 
griffe kann einen minimalen Standard für formverändernde Eingriffe am weiblichen Genitale sichern. Chirurgen ohne diese Erfahrungen sollten ihren Patientinnen nicht das Gefühl geben, dass es sich bei der Verkleinerung der kleinen Labien um einen allgemein anerkannten Routineeingriff handelt (Goodman 2009).

Auch wenn sich eines Tages Standards für Operationen im Genitalbereich durchgesetzt haben werden, wird ein individuelles Restrisiko für jede Patientin verbleiben.

\section{Literatur}

Alter G) (1998) A new technique for aesthetic labia minora reduction. Ann Plast Surg 40, 287-290 Alter GI (2006) Aesthetic Genital Surgery. In: Mathes SI (Hrsg.) Plastic Surgery. 404-410. Elsevier Philadelphia Borkenhagen A, Brähler E, Kentenich H (2009) Intimchirurgie: Ein gefährlicher Trend. Deutsches Ärzteblatt 106(11), A-500/B-430/C-416

Borkenhagen A, Kentenich H (2009) Labienreduktion - Neuester Trend der kosmetischen Genitalkorrektur Übersichtsarbeit. Geburtsh Frauenheilk 69, 1-5

Bravo Dr.-Sommer-Studie (2009) Liebe! Körper! Sexualität! Bauer München

Bravo Dr.-Sommer-Studie (2006) Liebe! Körper! Sexualität! Bauer München

Breitmeier 0 (2006) Plastische Vulva-Operationen. FRAUENARZT 47(6), 498-499

Caparo VI (1971) Congenital anomalies. Clin Obstet Gynecol 14, 988

Choi HY, Kim KT (2000) A new method for aesthetic reduction of the labia minora. Plastic Reconstr Surg 105, 419-422

Deutsche Gesellschaft für Gynäkologie und Geburtshilfe e.V. (2009) Stellungnahme zur Intimchirurgie. Online unter: http://www.dggg.de/uploads/media/pm_140709_icp.pdf (abgerufen am 02.10.2013)

Ellsworth WA, Rizvi M, Lypka M, Gaon M, Smith B, Cohen B, Dinh T (2010) Techniques for Labia Minora Reduction: An Algorithmic Approach. Aesth Plast Surg 34, 105-110

Giraldo F, González C, Fabiola de Haro MD (2004) Central wedge nymphectomy with a 90-degree z-plasty for aesthetic reduction of the labia minora. Plast Reconstr Surg 113, 1820-1825

Gress S (2011) Form- und Funktionsverbessernde Eingriffe im weiblichen Genitalbereich. In: Heimburg D von, Lemperle G (Hrsg.) Ästhetische Chirurgie. 25. Erg Lfg 4/11; XI-5. Ecomed Medizin Heidelberg

Gress S (2007) Aesthetic and functional corrections of the female genital area. Gynäkologisch Geburtshilfliche Rundschau 47, 23-32

Goodman MP (2009) Female cosmetic genital surgery. Obstet Gynecol 113(1), 154-159

Goodman MP, Placik OJ, Benson RH III, Miklos JR, Moore RD, Jason RA, Matlock DL, Simopoulos AF, Stern BH, Stanton RA, Kolb SE, Gonzalez F (2010) A large multicenter outcome study of female genital plastic surgery. I Sex Med 7, 165-177

Goodman MP (2011) Female Genital Cosmetic and Plastic Surgery: A Review. I Sex Med 8(6), 1813-1825

Hohl MK, Scheidel HP, Schüssler B (2008) Designer Vagina. Frauenheilkunde Aktuell 01/08, 4-13

Hwang W, Chang T, Sun P, Shung T (1983) Vaginal reconstruction using labia minora flaps in congenital total absence. Ann Plast Surg 18, 534

Koning M, Zeijlmans IA, Bouman TK, van der Lei B (2009) Female attitudes regarding labia minora appearance and reduction with consideration of media influence. Aesthet Surg | 29(1), 65-71

Lenzen-Schulte M (2009) Schönheitschirurgie. Riskante Manipulationen. Online unter: http://www.faz.net/ aktuell/wissen/mensch-gene/schoenheitschirurgie-riskante-manipulationen-1813515.html (abgerufen am 02.10.2013)

Liao LM, Creighton SM (2007) Requests for cosmetic genitoplasty: how should healthcare providers respond? British Medical Journal 334, 1090-1092 
Lloyd I, Crouch NS, Minto CL, Liao LM, Creighton SM (2005) Female genital appearance: "normality" unfolds. BJOG 112(5), 643-646

Maas SM, Hage II (2000) Functional and aesthetic labia minora reduction. Plast Reconstr Surg 105, 1453-1456

Medalie DA (2010) Aesthetic Female Genital Surgery. In: Siemionow MZ, Eisenmann-Klein M (Hrsg.) Plastic and Reconstructive Surgery. 687-692. Springer-Verlag London

Mirzabeigi MN, Moore JH Ir, Mericli AF, Bucciarelli P, Jandali S, Valerio IL, Stofman GM (2012) Current trends in vaginal labioplasty: a survey of plastic surgeons. Ann Plast Surg 68(2), 125-134

Munhoz AM (2006) Aesthetic labia minora reduction with. inferior wedge resection and superior pedicle flap reconstruction. Plast Reconstr Surg 118, 1237-1247

Paarlberg M, Weijenborg P (2008) Request for operativ reduction of the labia minora: a proposal for a practical guideline for gynecologists. I of Psychosom Obstetrics and Gynecology 29, 230-234

Pardo |, Sola V, Ricci P, Guilloff E (2006) Laser labioplasty of the labia minora. Int I Gynaecol Obstet 93, 38-43

Piza-Katzer H, Balogh B (2007) Ästhetische Chirurgie des äußeren Genitales. In: Berger A, Hierner R (Hrsg.) Plastische Chirurgie, Band III: Mamma-Stamm-Genitale. 378. Springer-Verlag Berlin Heidelberg

Ricci JV (1949) The Developement of Gynaecological Surgery and Instruments. The Blakiston Company Philadelphia Toronto

Rouzier R, Louis-Sylvestre C, Paniel B], Haddad B (2000) Hypertrophy of labia minora: Experience with 163 reductions. Am I Obstet Gynecol 182, 35-40

Schenda R (1998) Gut bei Leibe. Hundert wahre Geschichten vom menschlichen Körper. Beck München

Stadt Wien (2010) Weibliche Genitalchirurgie. Online unter: http://www.frauengesundheit-wien.at/ frauengesundheit/weibliche_genitalchirurgie.html (abgerufen am 02.10.2013)

Siemionow MZ, Eisenmann-Klein M (Hrsg.) (2010) Plastic and Reconstructive Surgery. Springer-Verlag London

Solanki NS, Tejero-Trujeque R, Stevens-King A, Malata CM (2009) Aesthetic and functional reduction of the labia minora using the Maas and Hage technique. I plast reconstr aest surg 63, 1181-1185

Turkof E, Sonnleitner E, Stirn A (2008) Schamlippenkorrektur. Wilhelm Maudrich Verlag Wien

Wild V, Poulin H, Biller-Andorno N (2009) Rekonstruktion des Hymens. Zur Ethik eines tabuisierten Eingriffs. Dt Ärzteblatt 106/8, 340-341 